\title{
Corynebacterium tuberculostearicum
}

National Cancer Institute

\section{Source}

National Cancer Institute. Corynebacterium tuberculostearicum. NCI Thesaurus. Code C114249.

A species of aerobic, Gram positive, rod shaped bacteria assigned to the phylum Actinobacteria. This bacteria is non-motile, non-spore forming, lipophilic, catalase positive, produces tuberculostearic acid, reduces nitrate and ferments saccharose. C. tuberculostearicum is a part of the normal human skin flora, but may also be involved in the development of inflammatory breast diseases. 